\title{
Detrimental Effects of Different Isolates of Beauveria bassiana on Developmental Profile of Spodoptera litura
}

\author{
Harikesh Singh ${ }^{1}$ and Wajid Hasan ${ }^{2 *}$ \\ ${ }^{1}$ Department of Entomology, Gochar Mahavidyalaya, Rampur Maniharan, \\ Saharanpur (U.P.), India \\ ${ }^{2}$ Krishi Vigyan Kendra, Jehanabad, Bihar, India \\ *Corresponding author
}

A B S T R A C T

\section{Keywords \\ Spodoptera litura, Beauveria bassiana, Developmental profile, Success index. \\ Article Info \\ Accepted: \\ 26 June 2017 \\ Available Online: \\ 10 July 2017}

Four isolates of Beauveria bassiana were tested against two age groups i.e. 0-24 hr and six day old larvae of Spodoptera litura by direct topical methods and crawling on treated leaves to observe the effect on growth and development. Six concentrations of each isolate were used against both the age groups and both the methods of application. Larval and pupal periods of neonate and 6 day old larvae were not affected by all isolates. However, percent pupation and adult emergence were significantly decreased in all isolates of $B$. bassiana at $10^{6}$ to $10^{8}$ spores per $\mathrm{ml}$ when applied on neonate larvae while low percent pupation and adult emergence were recorded only at $10^{8}$ spores per $\mathrm{ml}$ when applied by both the methods.

\section{Introduction}

Spodoptera litura (Fabricius) is a widely distributed South East Asia, feeding on 63 plant species belonging to 22 families (Prasad and Bhattacharya, 1975). It is a serious pest of soybean, pulses, oilseeds, cotton and vegetables (Bhattacharya and Rathore, 1977; Seema Rani et al., 2002). In an outbreak phase this insect can completely defoliate large area of crops causing reduction in yield. About 70 percent avoidable loss has been estimated due to this pest in blackgram (Krishnaiah et al., 1983). Heavy use of synthetic organic insecticides to control this pest resulted in development of resistance against insecticides belongs to different groups (Ramakrishnan et al., 1984;
Muregesan and Dhingra, 1995). Therefore, alternative control measures were searched out for this noxious pest. In recent years Biointensive Integrated Pest Management is emphasized.

Use of ecofriendly approach with entomopathogenic fungi, bacteria, virus and nematodes may provide a stable pest management programme (Jayraj, 1985).

Among the microbial pathogens of insects, entomopathogenic fungi are a very important group which can flourish in water, soil and air (Ferron, 1985). Therefore the experiment was carried out to observe the effects of four 
isolates of Beauveria bassiana on the growth and development.

\section{Materials and Methods}

\section{Fungal isolates and their culture}

Four fungal isolates were used in this investigation (Table 1). These isolates were grown on potato dextrose agar medium in 500 $\mathrm{ml}$ flasks at $25 \pm 1{ }^{\circ} \mathrm{C}$. Spores from these cultures were sprayed on S. litura and again isolated from dead insect to maintain virulence. For experiments conidia were harvested from 20 days old fungal culture by washing the surface with $50 \mathrm{ml}$ sterilized, distilled water containing 0.05 percent Tween 80 under aseptic condition. Concentration of spore was measured by haemocytometer under a microscope. Different concentrations of spore suspension were prepared in water having Tween 80 by serial dilution (Table 1 ).

This experiment was conducted on two age groups, neonate (0-24 h old) and 6 days old larvae, to observe the potency of four isolates of $B$. bassiana. Larvae for experiment were obtained from the nucleus culture. Spore suspension was directly applied on test insect and also on castor leaves followed by crawling of insect on treated leaves. Six concentrations of each isolate were used (Table 2). Sterilized distilled water containing Tween 80 (0.05 percent) was used as control. One $\mathrm{ml}$ of spore suspension of each concentration was sprayed on both sides of castor leaf or on 10 larvae with the help of Potter's tower at 20 psi pressure. Neonate larvae were initially reared in groups of 10 in plastic vial $(3 \times 10 \mathrm{~cm})$ upto 6 days after which they were reared individually. However, 6 days old larvae were reared individually from start of the experiment. These vials were provided with screw cap fitted with brass wire mesh for free aeration. Castor leaves were first changed after $48 \mathrm{~h}$ of spraying the spore suspension and thereafter fresh leaves were provided ad libitum. Sterilized sand was provided at the bottom of the vials to fully grown larvae to facilitate pupation. Each treatment was replicated three times and 10 insects were used in each replication. Observations were recorded on mortality at $24 \mathrm{~h}$ interval. Surviving larvae of both age groups and methods of application were allowed to complete the development. Observations were recorded on larval period, pupal period, pupation and adults' emergence for all four isolates. From these observations Growth index (Pant, 1956) and Success index (Prasad and Bhattacharya, 1975) were also calculated as follows:

Growth index $(\mathrm{GI})=\frac{\mathrm{N}}{\mathrm{Av}}$

Where,

$\mathrm{N} \quad=$ percent adult emergence

Av = average developmental period

Success index $=\frac{\text { LPI }+ \text { PPI }+ \text { PI }+ \text { AEI }}{4}$

Where,

Larval period index $(\mathrm{LPI})=$

\begin{tabular}{ccc} 
Larval period in control & \\
\hline Larval period in treatme nt
\end{tabular}

Pupal period index $(\mathrm{PPI})=$

\begin{tabular}{ccc} 
Pupal period in control \\
\hline$P$ upal period in treatme nt
\end{tabular}

Pupation index (PI) =

Percent pupation in treatme nt

Percent pupation in control

Adult emergence index $(\mathrm{AEI})=$

\begin{tabular}{cccc} 
Percent adult emergence in treatme nt \\
\hline Percent adult emergence in control
\end{tabular}


The data on various developmental parameters were analysed under Complete Randomized Design (CRD) to compare the effect of different doses of $B$. bassiana. Angular transformation on percent pupation and adults' emergence were also analysed.

\section{Results and Discussion}

Surviving larvae of bioassay experiment were allowed to complete their development on castor leaves.

\section{Pantnagar isolate}

Figure 1 reveals that a marked decrease in larval survival was observed after 4 days of application of fungal spore by crawling method on neonate larvae. Larval survival decreased upto 10 days only at $6.1 \times 10^{6}$ to $6.1 \times 10^{8}$ spores per $\mathrm{ml}$ but beyond this period larval survival remained same. Dead larvae mummified with white fungal growth. No marked variation in larval and pupal periods were recorded but pupation was reduced significantly at $6.1 \times 10^{6}$ to $6.1 \times 10^{8}$ spores per ml. Adult emergence was further reduced at $6.1 \times 10^{5}$ and also at above concentration Growth and Success indices were lower in all fungal treatments as compared to untreated larvae feeding on castor leaves (Table 2).

Like crawling method, larval survival also decreased at $6.1 \times 10^{6}$ to $6.1 \times 10^{8}$ spores per ml when spores were applied topically on $0-24 \mathrm{~h}$ old larvae of $S$. litura (Figure 2). Pupation and adult emergence were significantly reduced at $6.1 \times 10^{5}$ and higher concentrations and it ranged from 33.3 to 93.3 percent. Low Growth and Success indices were also recorded in all test concentrations of Pantnagar isolate by topical application (Table 2).

Larval survival was reduced to 16.7 percent when Pantnagar isolate at $6.3 \times 10^{8}$ spores per $\mathrm{ml}$ was applied on 6 day old larvae of $S$. litura by above two methods. However, larval and pupal developmental periods were not affected by both the methods of application (Table 3). Pupation ranged from 83.3 to 100 percent in different concentrations by both methods but adult emergence was reduced to 76.7 percent by crawling method at $6.1 \times 10^{8}$ spores per $\mathrm{ml}$. Growth and Success indices fluctuated within a very narrow range for different concentrations when this isolate was applied by both methods on 6 day old larvae (Table 3).

\section{MTCC-984 isolate}

Larval mortality started in all test concentrations after 2 days of application of spores of MTCC-984 isolate by crawling method on neonate larvae. It gradually increased upto 10 days (Figure 3). However, larval and pupal periods were not affected by crawling method but pupation and adult emergence were significantly reduced at $5.9 \times$ $10^{4}$ to $5.9 \times 10^{8}$ spores per ml (Table 4$)$. A marked reduction in Growth and Success indices was apparent at $5.9 \times 10^{5}$ to $5.9 \times 10^{8}$ spores per $\mathrm{ml}$ on neonate larvae by crawling method.

Figure 4 indicates a reduction of 50 percent larval survival within 5 days of topical application of $5.9 \times 10^{8}$ spores per $\mathrm{ml}$ of MTCC-984 isolate on neonate larvae while in rest of the concentrations survival ranged from 70 to 90 percent. Developmental period was not affected but a significant reduction in pupation and adult emergence was recorded at $5.9 \times 10^{4}$ to $5.9 \times 10^{8}$ spore per $\mathrm{ml}$ when spores of this isolate were applied topically on neonate larvae (Table 4).

MTCC-984 reduced 6.7 to 16.7 percent larval survival by topical application and 13.3 to 30.0 percent by crawling method for 6 day old larvae when $6.0 \times 10^{7}$ and $6.1 \times 10^{8}$ spores per $\mathrm{ml}$, respectively, were used. No effect on larval and 
pupal periods was observed by this isolate of $B$. bassiana by either methods of application on 6 day old larvae of S. litura (Table 5). However, a little reduction in pupation and adult emergence were recorded at $6.0 \times 10^{7}$ and $6.0 \times 10^{8}$ spores per $\mathrm{ml}$ by crawling method and topical application, respectively.

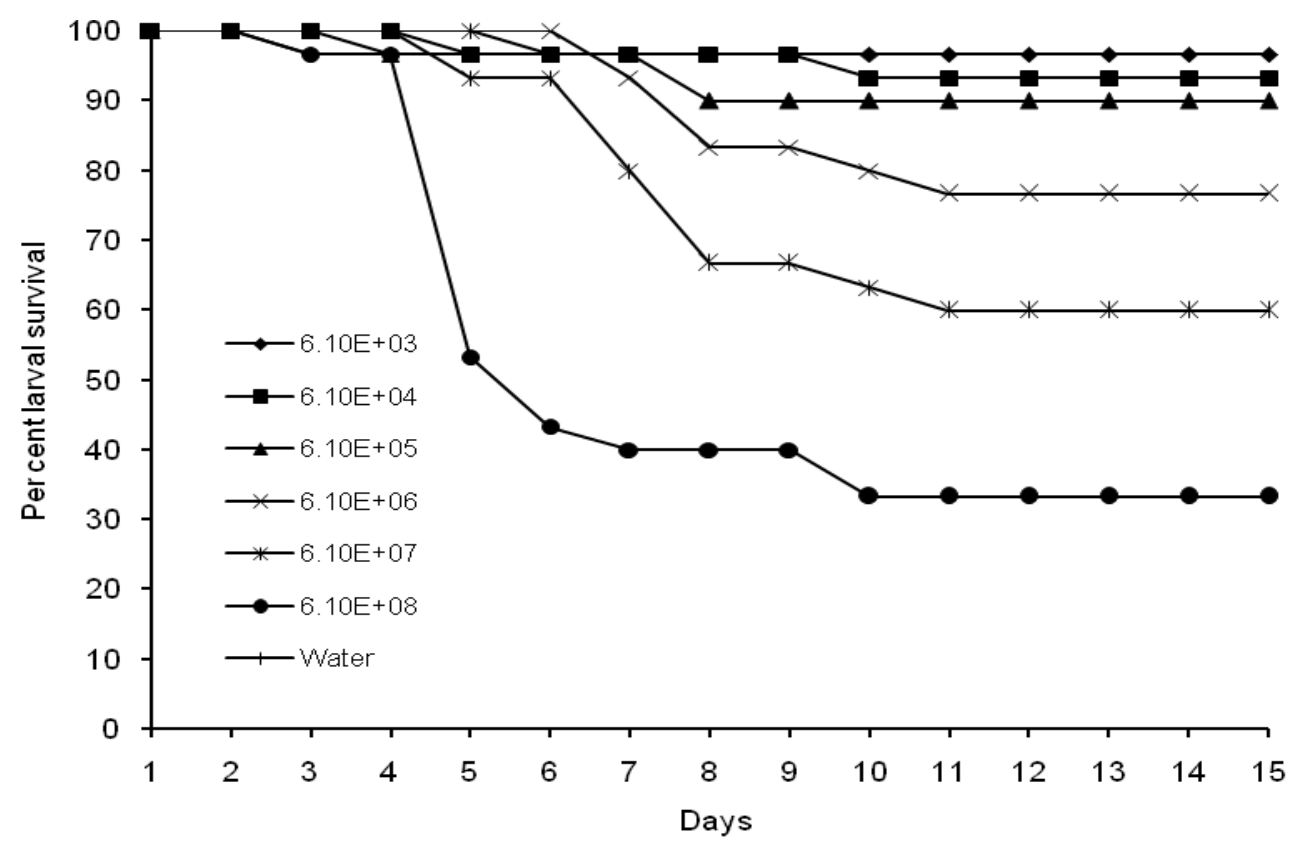

Figure 1. Larval survival of $S$. litura when $0-24 \mathrm{~h}$ old larvae were allowed crawl on castor leaves with different spore concentration of Pantnagar isolate of $B$. bassiana

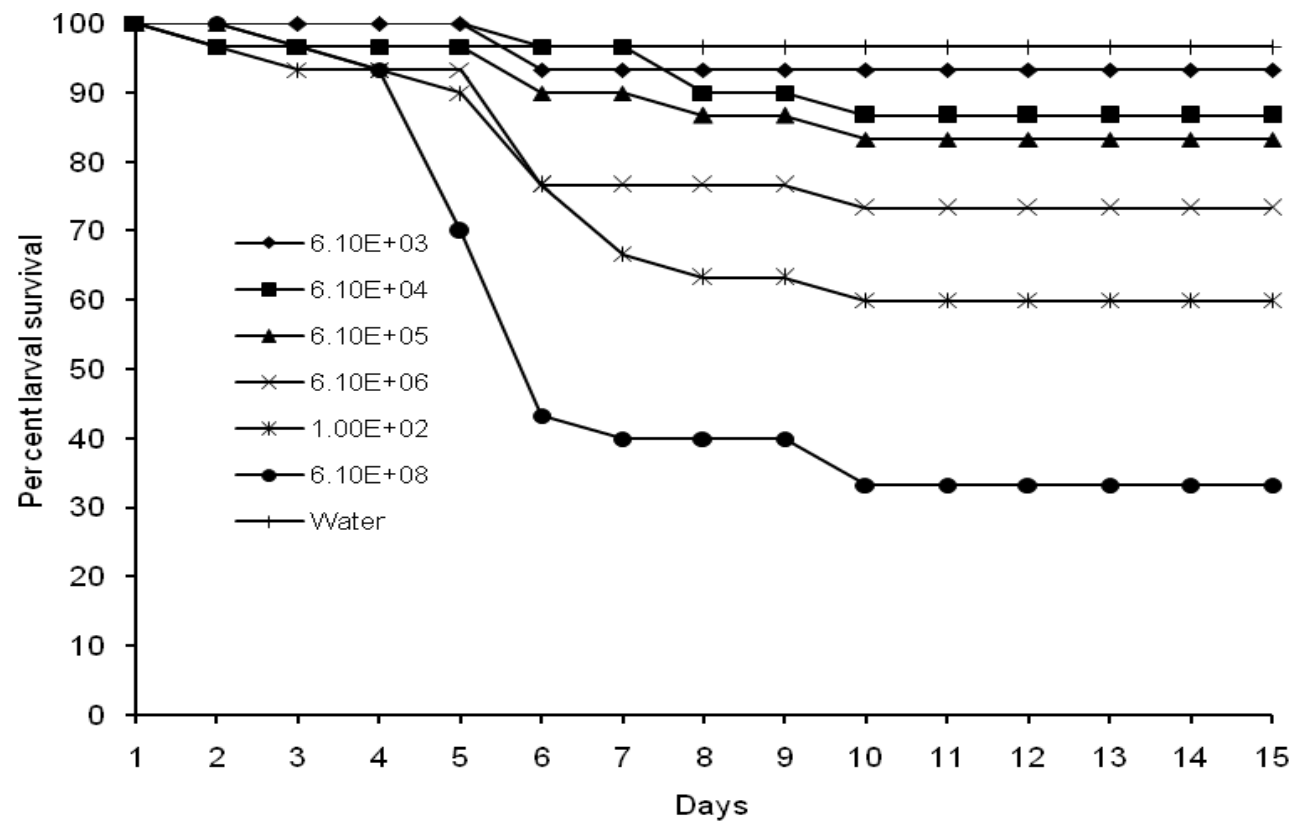

Figure 2. Larval survival of $S$. litura when different spore concentrations of Pantnagar isolate of $B$. bassiana were topically applied on 0-24 h larvae 


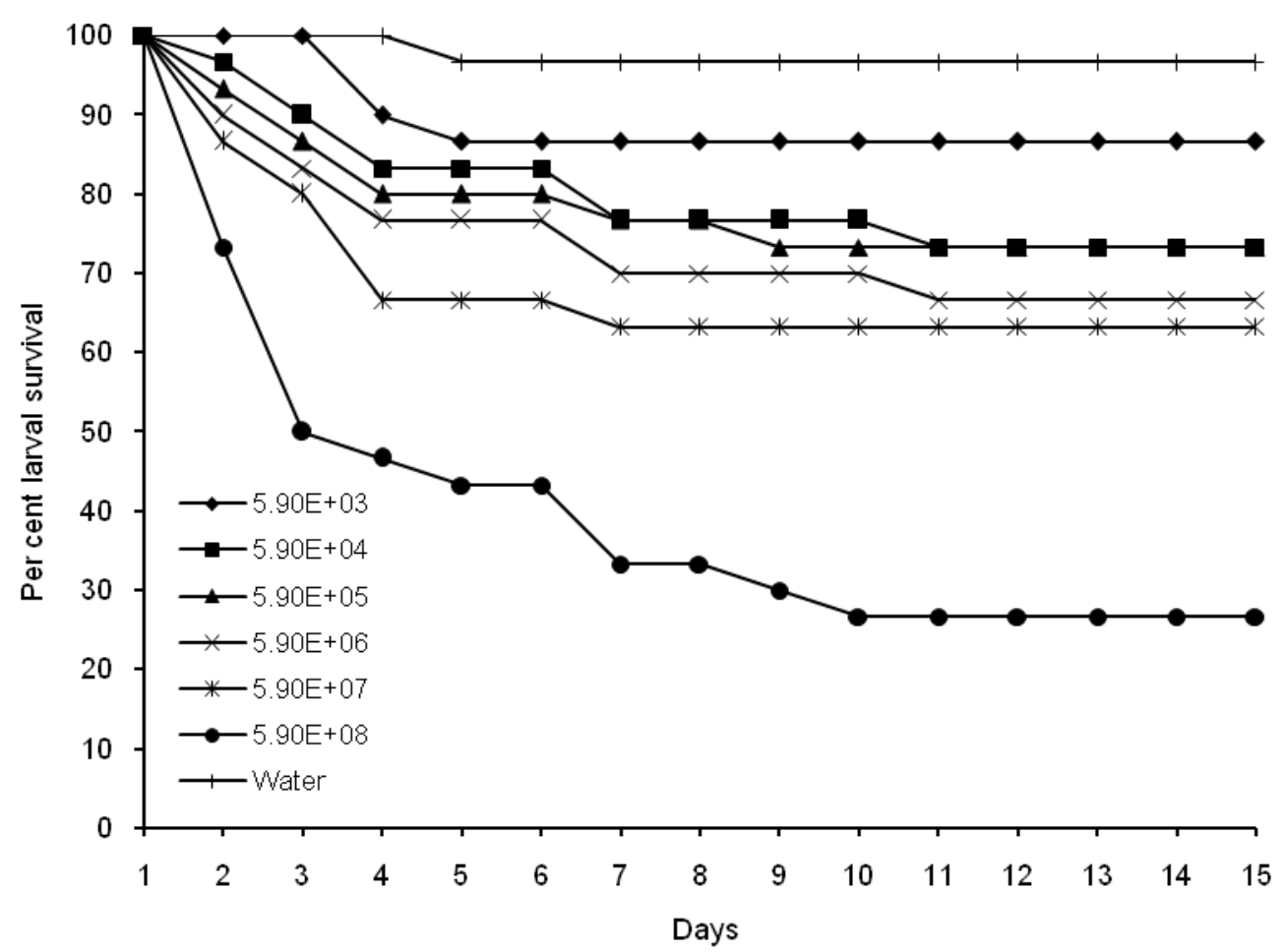

Figure 3. Larval survival of $S$. litura when 0-24 h old larvae were allowed crawl on castor leaves with different spore concentration of MTCC-984 isolate of $B$. bassiana

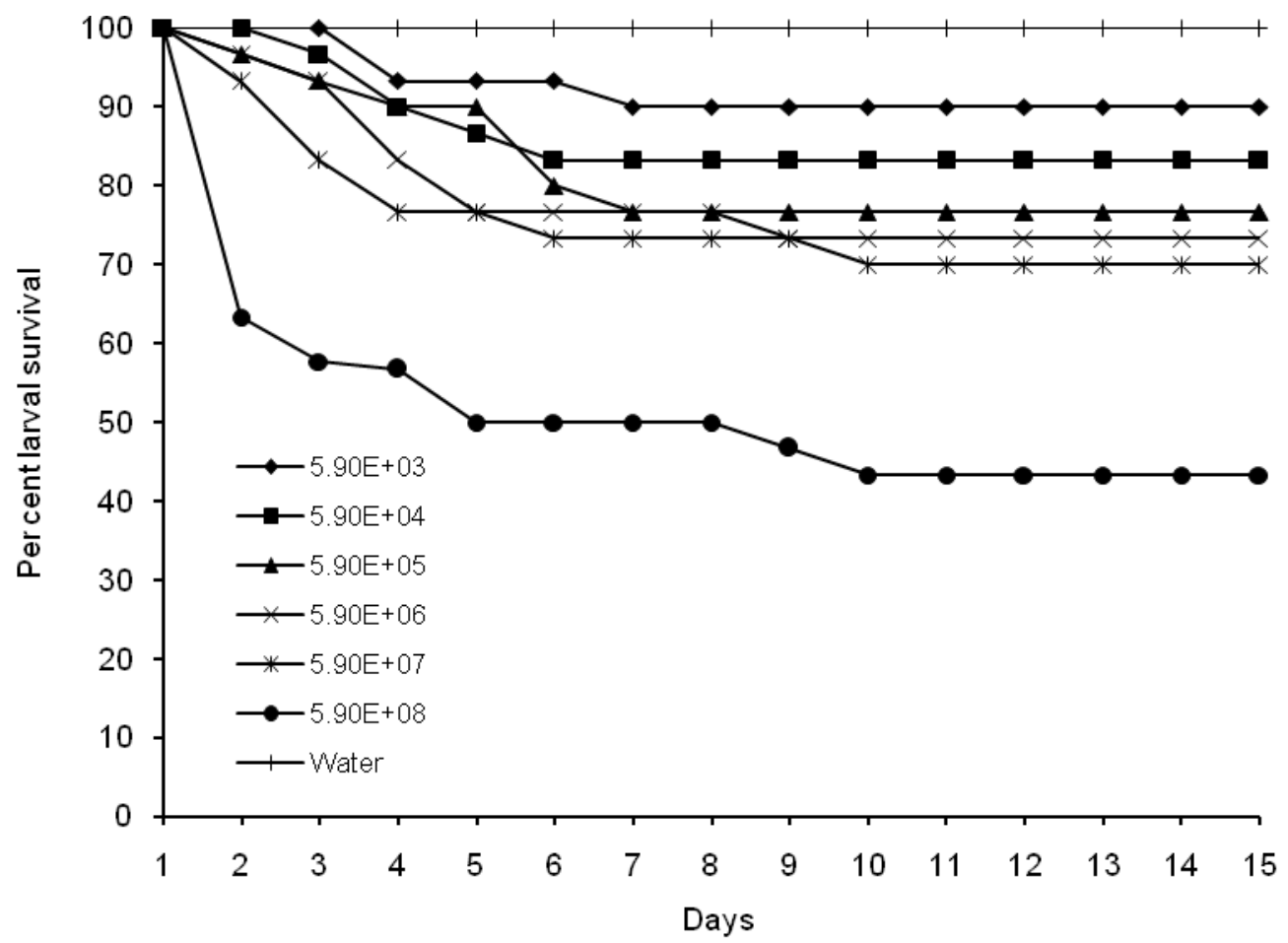

Figure 4. Larval survival of $S$. litura when different spore concentrations of MTCC-984 isolate of $B$. bassiana were topically applied on 0-24 h larvae 


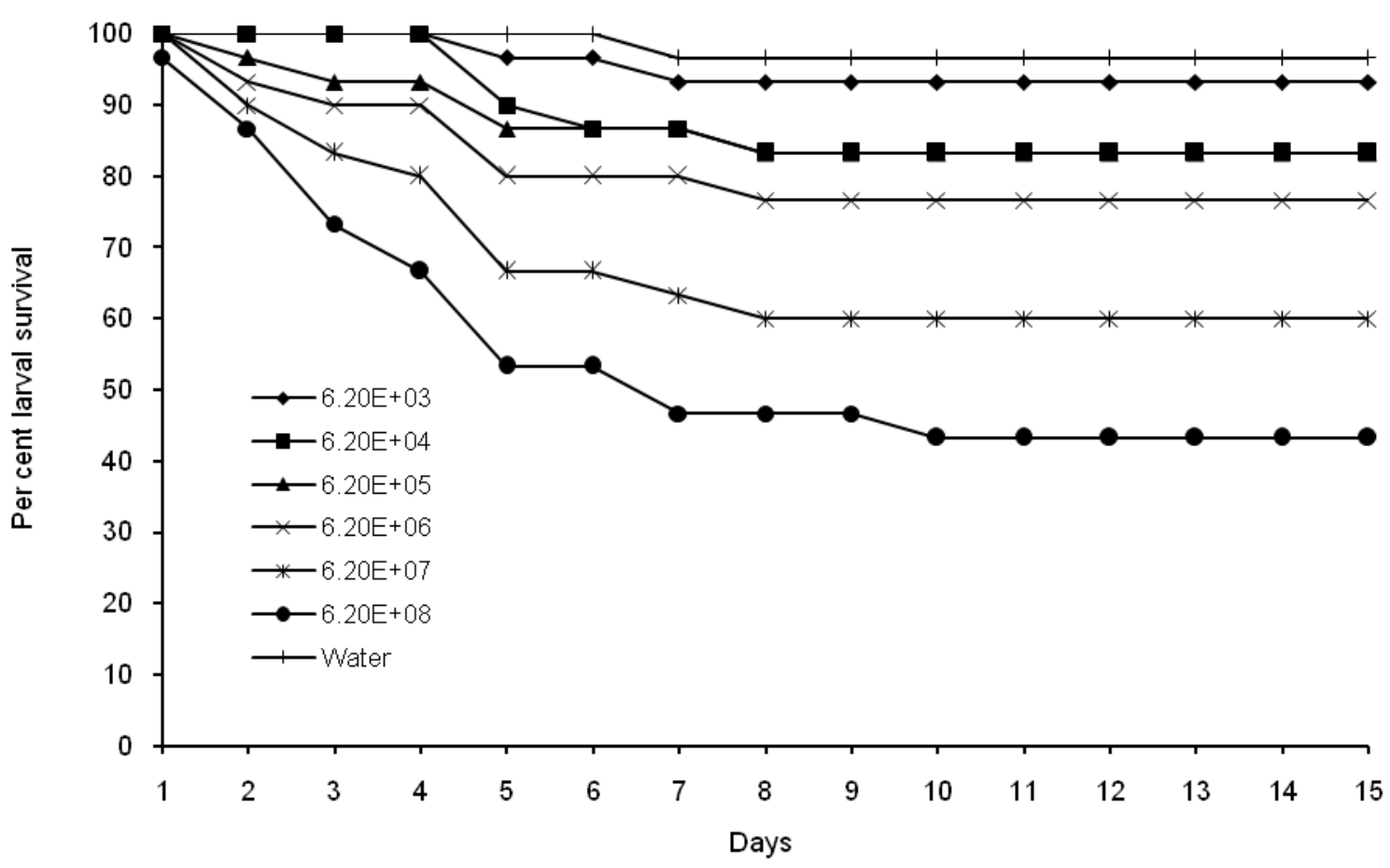

Figure 5. Larval survival of $S$. litura when $0-24 \mathrm{~h}$ old larvae were allowed crawl on castor leaves with different spore concentration of Chikmagalore isolate of $B$. bassiana

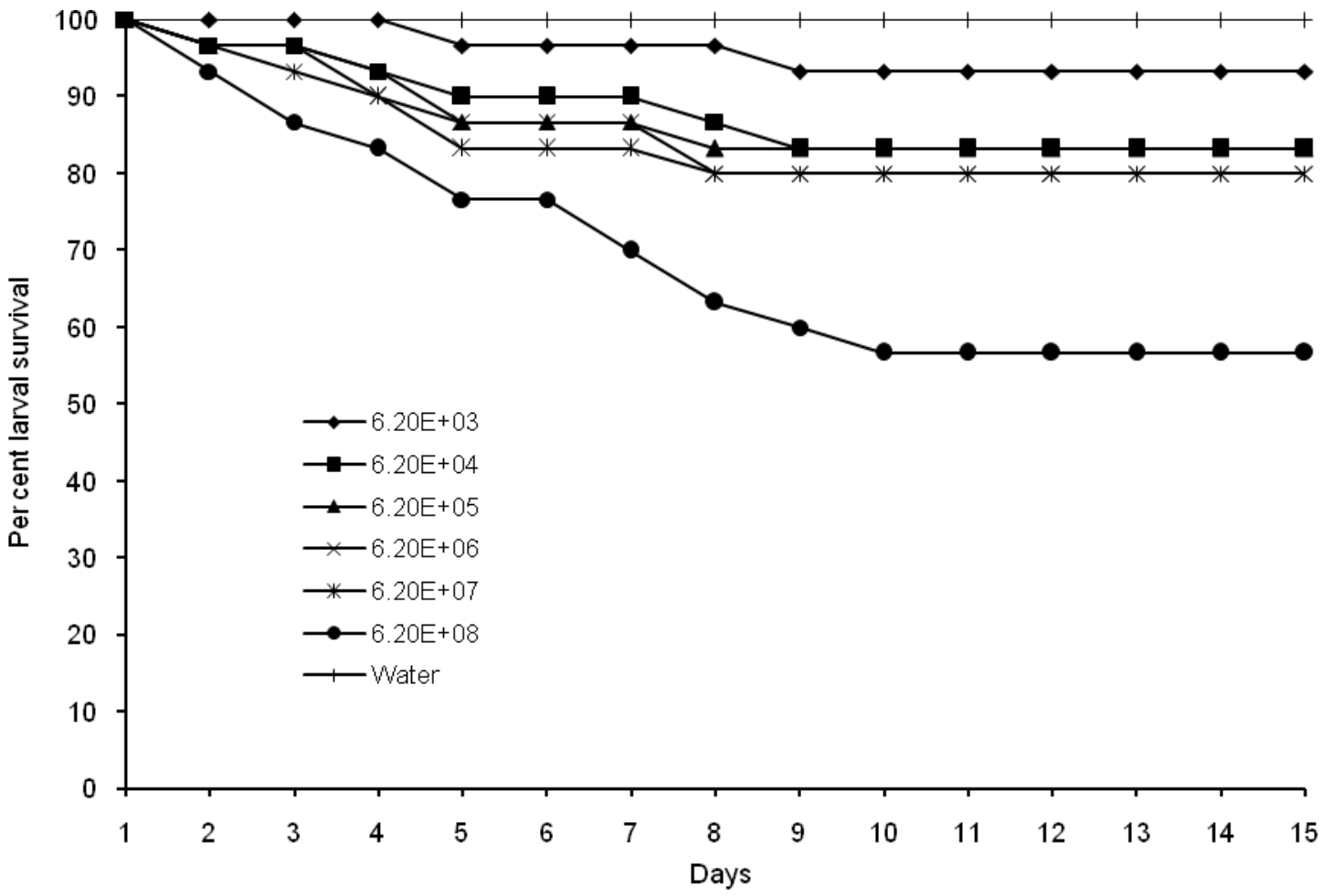

Figure 6. Larval survival of $S$. litura when different spore concentrations of Chikmagalore isolate of $B$. bassiana were topically applied on 0-24 h larvae 


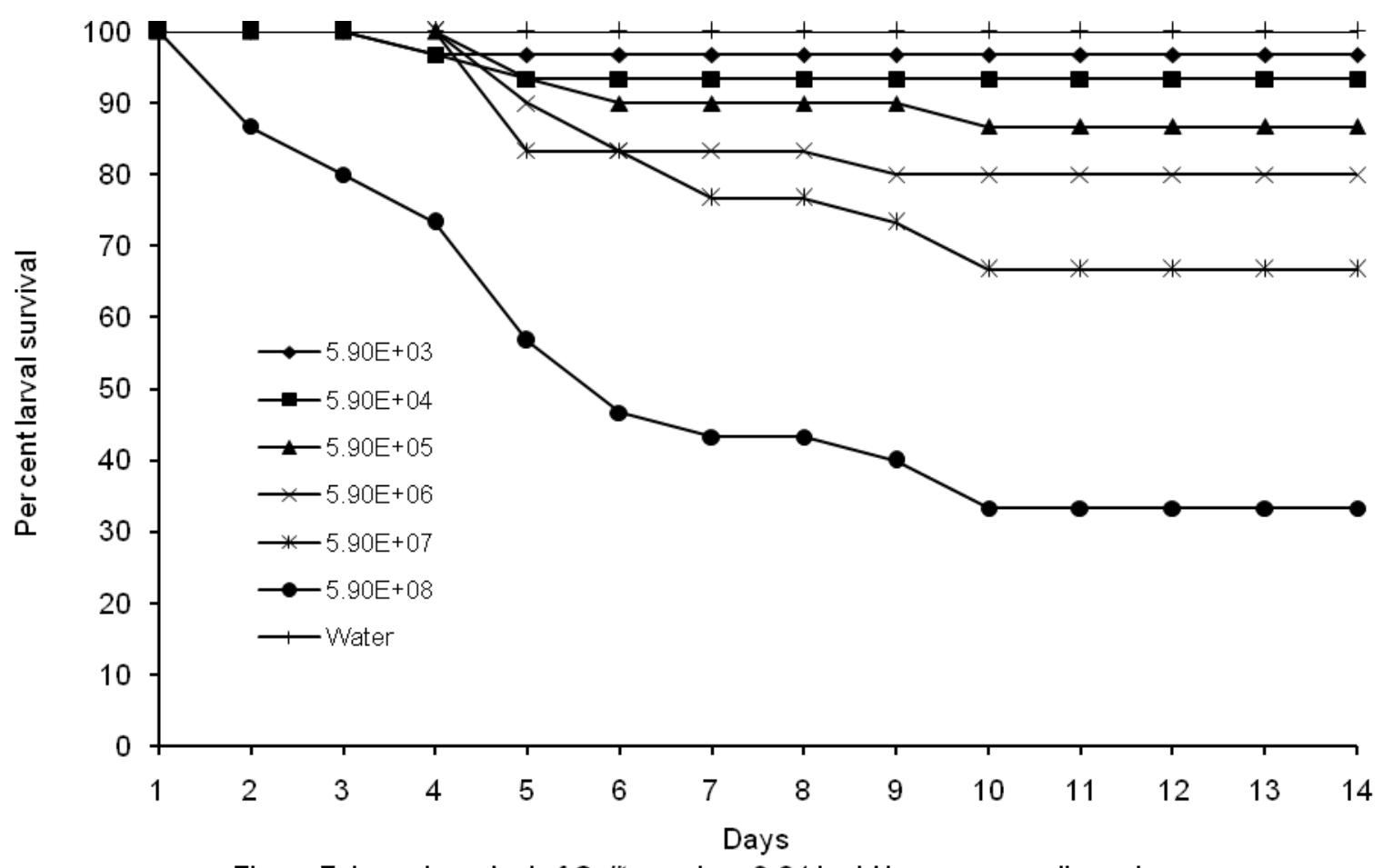

Figure 7. Larval survival of S. litura when 0-24 h old larvae were allowed crawl on castor leaves with different spore concentration of Delhi isolate of $B$. bassiana

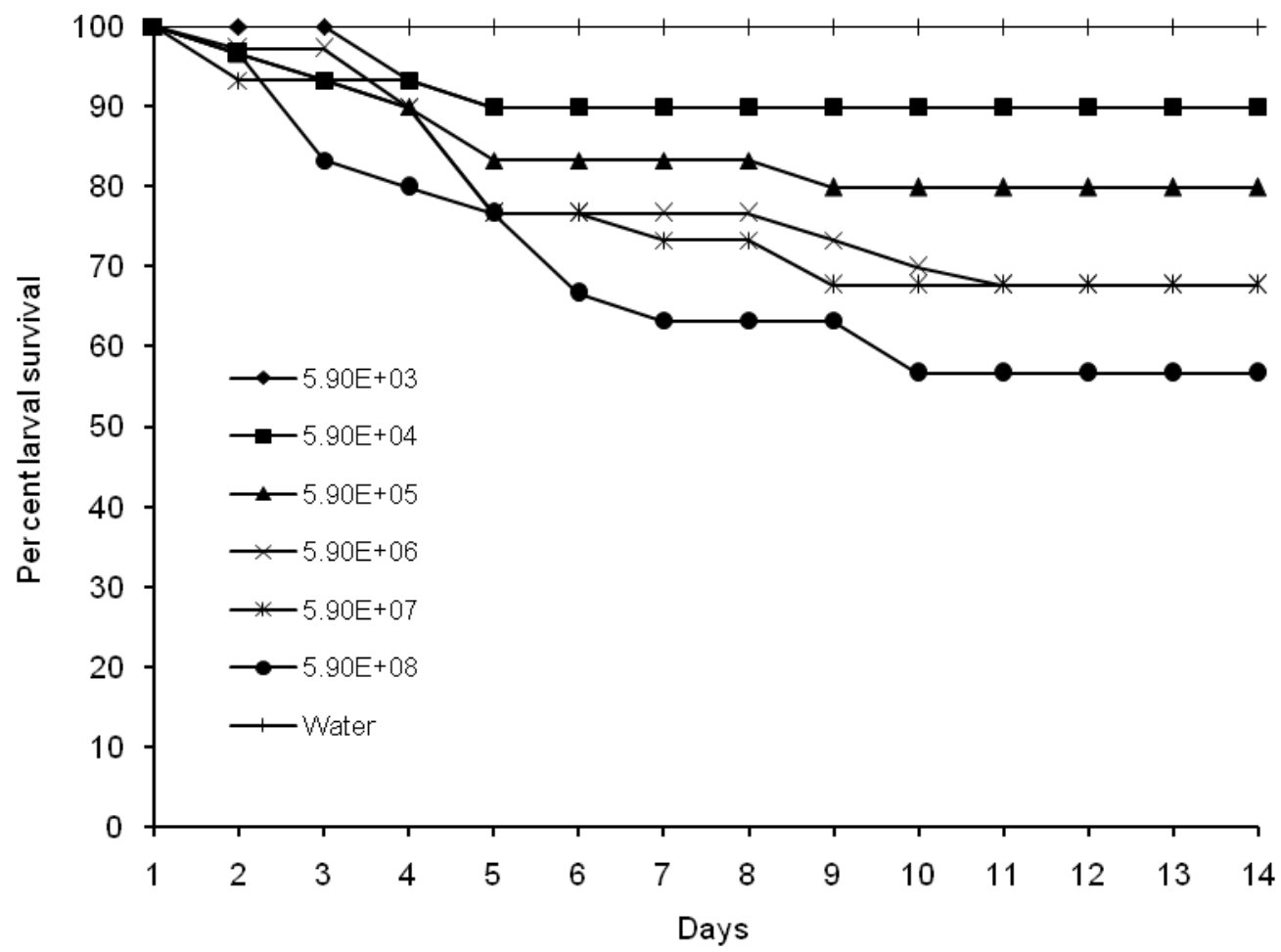

Figure 8. Larval survival of $S$. litura when different spore concentrations of Delhi isolate of $B$. bassiana were topically applied on 0-24 h larvae 
Table.1 Different isolates of B. bassiana, their sources and concentrations used for bioassay

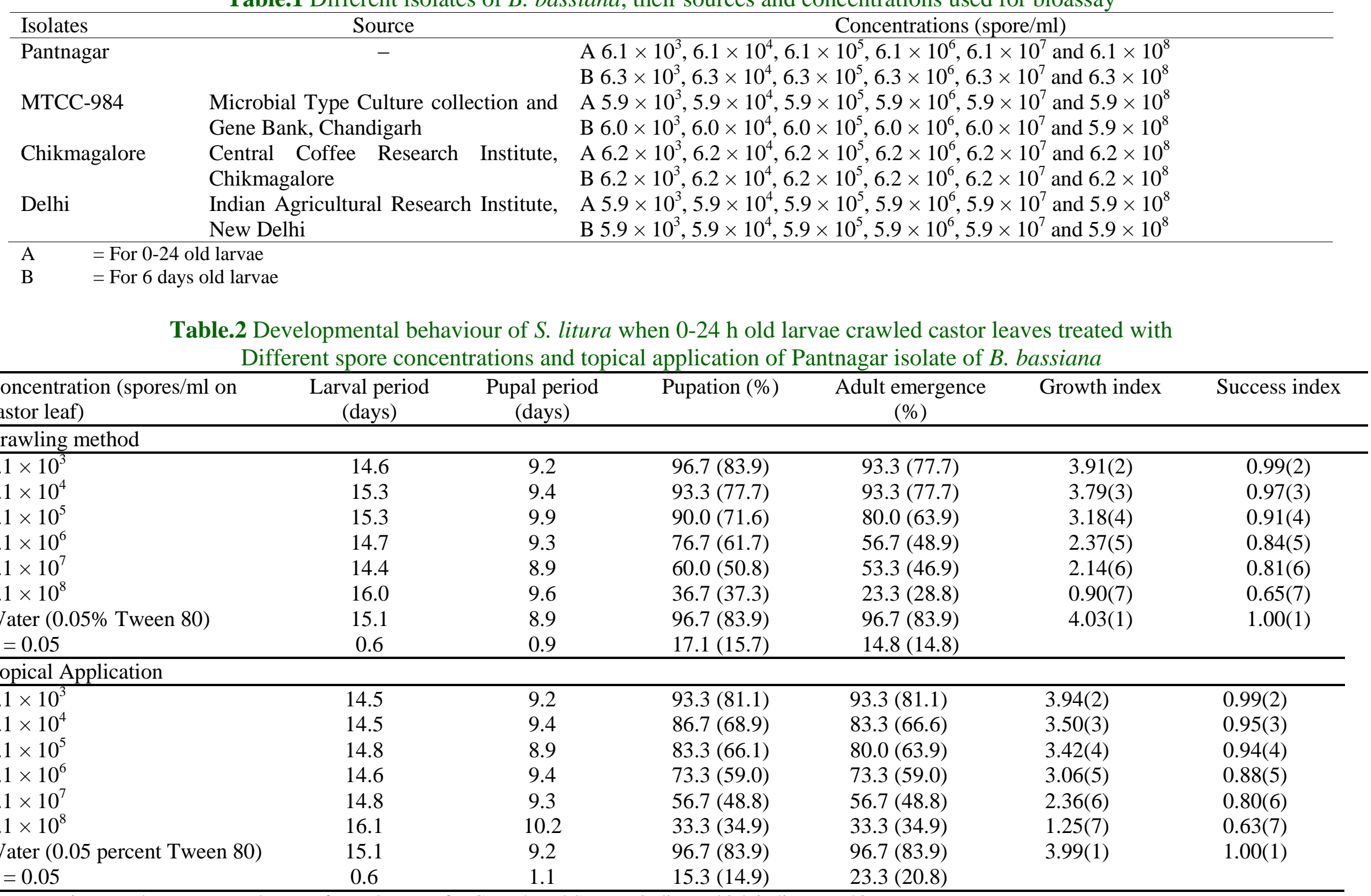

Data in parentheses are angular transformed except for Growth and Success indices which indicate ranking 
Table.3 Developmental behaviour of S. litura when 6 day old larvae crawled on castor leaves treated with different spore Concentrations and topical application of Pantnagar isolate of B. bassiana

\begin{tabular}{|c|c|c|c|c|c|c|}
\hline $\begin{array}{l}\text { Concentration (spores } / \mathrm{ml} \text { on } \\
\text { castor leaf) }\end{array}$ & $\begin{array}{l}\text { Larval period } \\
\text { (days) }\end{array}$ & $\begin{array}{l}\text { Pupal period } \\
\quad \text { (days) }\end{array}$ & Pupation (\%) & $\begin{array}{c}\text { Adult emergence } \\
(\%)\end{array}$ & Growth index & Success index \\
\hline \multicolumn{7}{|l|}{ Crawling Method } \\
\hline $6.3 \times 10^{3}$ & 14.7 & 8.4 & $100.0(90.0)$ & $100.0(90.0)$ & $4.33(2)$ & $0.98(2)$ \\
\hline $6.3 \times 10^{4}$ & 14.4 & 8.4 & $96.7(83.9)$ & $93.3(81.1)$ & $4.08(3)$ & $0.96(3)$ \\
\hline $6.3 \times 10^{5}$ & 14.4 & 9.1 & $93.3(77.7)$ & $93.3(77.7)$ & $3.97(4)$ & $0.94(4)$ \\
\hline $6.3 \times 10^{6}$ & 14.3 & 8.3 & $93.3(77.7)$ & $86.7(72.3)$ & $3.79(5)$ & $0.94(5)$ \\
\hline $6.3 \times 10^{7}$ & 14.3 & 8.9 & $90.0(71.6)$ & $83.3(66.1)$ & $3.59(6)$ & $0.91(6)$ \\
\hline $6.3 \times 10^{8}$ & 14.5 & 8.6 & $83.3(66.1)$ & $76.7(61.9)$ & $3.34(7)$ & $0.86(7)$ \\
\hline Water $(0.05$ percent Tween & 14.2 & 8.1 & $100.0(90.0)$ & $1.00(90.0)$ & $4.47(1)$ & $1.00(1)$ \\
\hline \multicolumn{7}{|l|}{ 80) } \\
\hline$P=0.05$ & 0.6 & 0.5 & $7.6(12.6)$ & $15.8(17.7)$ & & \\
\hline \multicolumn{7}{|l|}{ Topical Application } \\
\hline $6.3 \times 10^{3}$ & 14.4 & 8.7 & $100.0(90.0)$ & $100.0(90.0)$ & $4.33(2)$ & $0.99(2)$ \\
\hline $6.3 \times 10^{4}$ & 14.4 & 9.1 & $96.7(83.9)$ & $96.7(83.9)$ & $4.11(3)$ & $0.96(3)$ \\
\hline $6.3 \times 10^{5}$ & 14.4 & 8.3 & $93.3(77.7)$ & $90.0(75.0)$ & $3.96(4)$ & $0.96(3)$ \\
\hline $6.3 \times 10^{6}$ & 14.0 & 8.7 & $90.0(71.6)$ & $90.0(71.6)$ & $3.95(5)$ & $0.94(4)$ \\
\hline $6.3 \times 10^{7}$ & 14.3 & 9.3 & $90.0(75.0)$ & $86.7(68.9)$ & $3.67(6)$ & $0.91(5)$ \\
\hline $6.3 \times 10^{8}$ & 14.4 & 8.6 & $83.3(66.1)$ & $83.3(66.1)$ & $6.61(7)$ & $0.90(6)$ \\
\hline Water $(0.05$ percent Tween & 14.2 & 8.3 & $100.0(90.0)$ & $100(90.0)$ & $4.47(1)$ & $1.00(1)$ \\
\hline \multicolumn{7}{|l|}{ 80) } \\
\hline$P=0.05$ & 0.3 & 0.8 & $9.4(13.8)$ & $9.4(12.3)$ & & \\
\hline
\end{tabular}

Data in parentheses are angular transformed except for Growth and Success indices which indicate ranking 
Table.4 Developmental behaviour of S. litura when 0-24 h old larvae crowed on castor leaves treated with different spore Concentrations and topical application of MTCC-984 isolate of B. bassiana

\begin{tabular}{|c|c|c|c|c|c|c|}
\hline $\begin{array}{l}\text { Concentration (spores/ml on } \\
\text { castor leaf) }\end{array}$ & $\begin{array}{l}\text { Larval period } \\
\text { (days) }\end{array}$ & $\begin{array}{l}\text { Pupal period } \\
\text { (days) }\end{array}$ & Pupation (\%) & $\begin{array}{c}\text { Adult emergence } \\
(\%)\end{array}$ & Growth index & Success index \\
\hline \multicolumn{7}{|l|}{ Crawling Method } \\
\hline $5.9 \times 10^{3}$ & 15.6 & 7.4 & $86.7(68.9)$ & $86.7(68.9)$ & $3.78(2)$ & $0.95(2)$ \\
\hline $5.9 \times 10^{4}$ & 15.7 & 7.4 & $73.3(59.0)$ & $73.3(59.0)$ & $3.17(3)$ & $0.54(3)$ \\
\hline $5.9 \times 10^{5}$ & 16.1 & 7.4 & $73.3(59.0)$ & $70.0(56.8)$ & $2.98(4)$ & $0.86(4)$ \\
\hline $5.9 \times 10^{6}$ & 15.8 & 7.5 & $66.7(54.8)$ & $66.7(54.8)$ & $2.86(5)$ & $0.84(5)$ \\
\hline $5.9 \times 10^{7}$ & 16.2 & 7.5 & $63.3(52.8)$ & $63.3(52.8)$ & $2.67(6)$ & $0.82(6)$ \\
\hline $5.9 \times 10^{8}$ & 15.7 & 7.4 & $26.7(30.3)$ & $26.7(30.3)$ & $1.57(7)$ & $0.64(7)$ \\
\hline Water $(0.05$ percent Tween & 15.8 & 7.3 & $96.7(83.9)$ & $96.7(83.9)$ & $4.19(1)$ & $1.00(1)$ \\
\hline \multicolumn{7}{|l|}{ 80) } \\
\hline$P=0.05$ & 0.5 & 0.3 & $13.8(11.5)$ & $13.2(11.2)$ & & \\
\hline \multicolumn{7}{|l|}{ Topical Application } \\
\hline $5.9 \times 10^{3}$ & 15.7 & 7.6 & $90.0(71.6)$ & $90.0(71.6)$ & $3.86(2)$ & $0.94(2)$ \\
\hline $5.9 \times 10^{4}$ & 15.8 & 7.6 & $83.3(66.6)$ & $83.3(66.6)$ & $3.56(3)$ & $0.91(3)$ \\
\hline $5.9 \times 10^{5}$ & 15.8 & 7.4 & $73.3(59.2)$ & $73.3(59.2)$ & $3.16(4)$ & $0.87(4)$ \\
\hline $5.9 \times 10^{6}$ & 15.7 & 7.6 & $73.3(59.0)$ & $73.3(59.0)$ & $3.15(5)$ & $0.86(5)$ \\
\hline $5.9 \times 10^{7}$ & 15.7 & 7.6 & $70.0(56.8)$ & $70.0(56.8)$ & $3.00(6)$ & $0.84(6)$ \\
\hline $5.9 \times 10^{8}$ & 16.0 & 7.7 & $43.3(41.1)$ & $43.3(41.1)$ & $1.80(7)$ & $0.70(7)$ \\
\hline Water $(0.05$ percent Tween & 15.7 & 7.4 & $100.0(90.0)$ & $100.0(90.0)$ & $4.33(1)$ & $1.00(1)$ \\
\hline \multicolumn{7}{|l|}{ 80) } \\
\hline$P=0.05$ & 0.5 & 0.2 & $13.8(9.1)$ & $13.8(9.1)$ & & \\
\hline
\end{tabular}

Data in parentheses are angular transformed except for Growth and Success indices which indicate ranking 
Int.J.Curr.Microbiol.App.Sci (2017) 6(7): 2378-2394

Table.5 Developmental behaviour of $S$. litura when 6 day old larvae crawled on castor leaves treated with different spore Concentrations and topical application of MTCC-984 isolate of B. bassiana

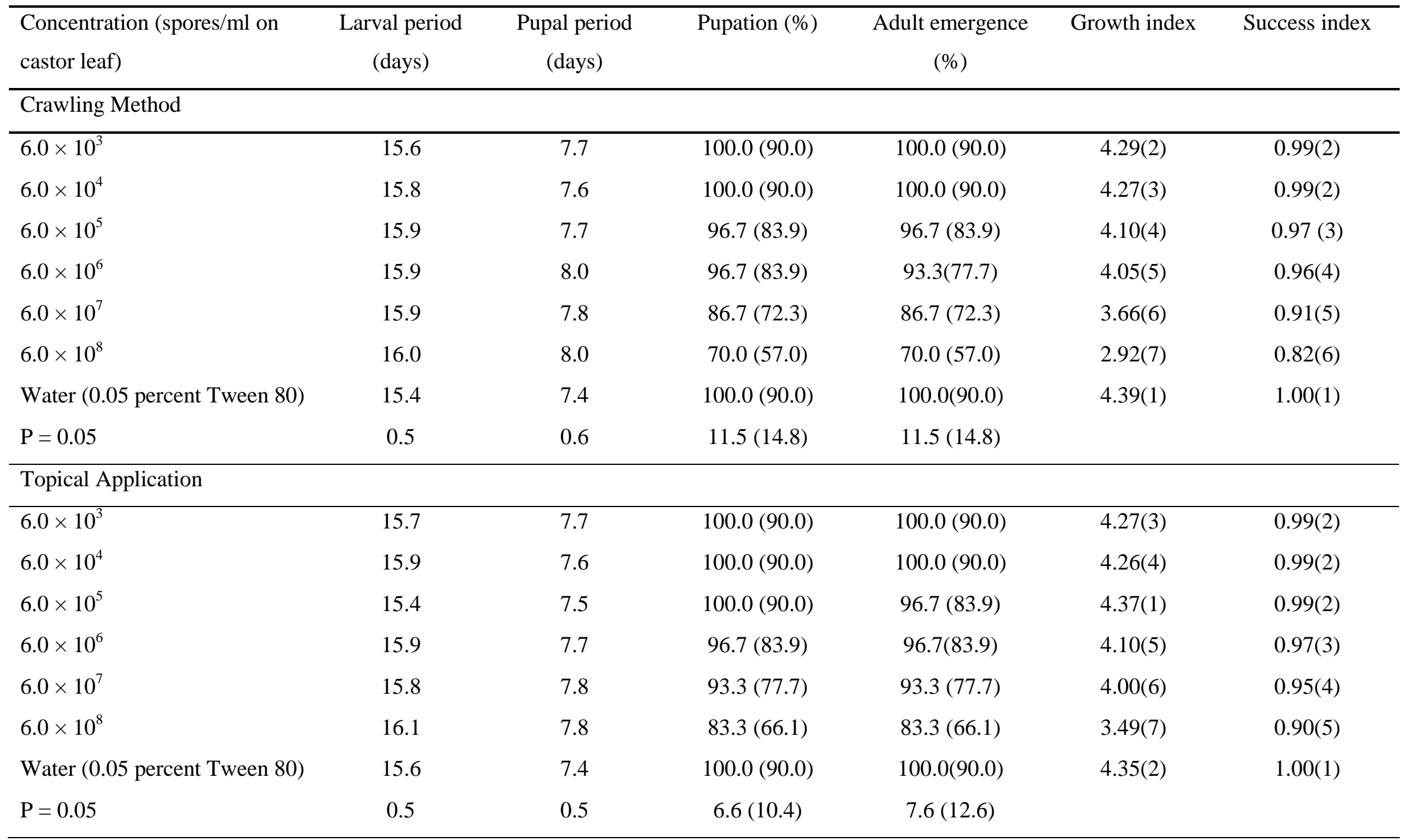

Data in parentheses are angular transformed except for Growth and Success indices which indicate ranking 
Table.6 Developmental behaviour of $S$. litura when 0-24 h old larvae crawled castor leaves treated with

Different spore concentrations and topical application of Chikmagalore isolate of B. bassiana

\begin{tabular}{|c|c|c|c|c|c|c|}
\hline $\begin{array}{l}\text { Concentration (spores/ml on } \\
\text { castor leaf) }\end{array}$ & $\begin{array}{l}\text { Larval period } \\
\text { (days) }\end{array}$ & $\begin{array}{l}\text { Pupal period } \\
\quad \text { (days) }\end{array}$ & Pupation (\%) & $\begin{array}{l}\text { Adult emergence } \\
(\%)\end{array}$ & Growth index & Success index \\
\hline \multicolumn{7}{|l|}{ Crawling Method } \\
\hline $6.2 \times 10^{4}$ & 15.8 & 7.6 & $83.3(66.1)$ & $83.3(66.1)$ & $3.56(3)$ & $0.93(3)$ \\
\hline $6.2 \times 10^{5}$ & 16.3 & 7.8 & $83.3(66.1)$ & $83.3(66.1)$ & $3.46(4)$ & $0.91(5)$ \\
\hline $6.2 \times 10^{7}$ & 15.9 & 8.0 & $60.0(50.8)$ & $60.0(50.8)$ & $2.61(6)$ & $0.89(6)$ \\
\hline $6.2 \times 10^{8}$ & 16.3 & 7.9 & $43.3(41.2)$ & $43.3(41.2)$ & $1.79(7)$ & $0.70(7)$ \\
\hline Water $(0.05$ percent Tween & 15.5 & 7.6 & $96.7(83.9)$ & $96.7(83.9)$ & $4.19(1)$ & $1.00(1)$ \\
\hline \multicolumn{7}{|l|}{ 80) } \\
\hline $6.2 \times 10^{4}$ & 15.7 & 7.7 & $83.3(66.1)$ & $83.3(66.1)$ & $3.56(3)$ & $0.91(3)$ \\
\hline $6.2 \times 10^{5}$ & 15.7 & 7.8 & $83.3(66.1)$ & $83.3(66.1)$ & $3.54(4)$ & $0.91(3)$ \\
\hline $6.2 \times 10^{6}$ & 15.9 & 7.8 & $80.0(67.9)$ & 76.7(65.9) & $3.24(6)$ & $0.87(5)$ \\
\hline $6.2 \times 10^{7}$ & 16.1 & 7.4 & $80.0(63.9)$ & $76.7(61.2)$ & $3.26(5)$ & $0.89(4)$ \\
\hline $6.2 \times 10^{8}$ & 16.2 & 7.8 & $43.3(41.2)$ & $43.3(41.2)$ & $1.80(7)$ & $0.70(6)$ \\
\hline Water $(0.05$ percent Tween & 15.5 & 7.6 & $100.0(90.0)$ & $100.0(90.0)$ & $4.33(1)$ & $1.00(1)$ \\
\hline \multicolumn{7}{|l|}{ 80) } \\
\hline$P=0.05$ & 0.7 & 0.4 & $16.2(17.0)$ & $17.1(17.5)$ & & \\
\hline
\end{tabular}

Data in parentheses are angular transformed except for Growth and Success indices which indicate ranking 
Table.7 Developmental behaviour of $S$. litura when 6 day old larvae crawled castor leaves treated with Different spore concentrations of Chikmagalore isolate of $B$. bassiana

\begin{tabular}{|c|c|c|c|c|c|c|}
\hline $\begin{array}{l}\text { Concentration (spores/ml on } \\
\text { castor leaf) }\end{array}$ & $\begin{array}{l}\text { Larval period } \\
\text { (days) }\end{array}$ & $\begin{array}{l}\text { Pupal period } \\
\text { (days) }\end{array}$ & Pupation (\%) & $\begin{array}{c}\text { Adult emergence } \\
(\%)\end{array}$ & Growth index & Success index \\
\hline \multicolumn{7}{|l|}{ Crawling Method } \\
\hline $6.2 \times 10^{3}$ & 15.9 & 7.9 & $96.7(83.9)$ & $96.7(83.9)$ & $4.06(5$ & $0.958(5)$ \\
\hline $6.2 \times 10^{4}$ & 15.8 & 7.6 & $100.0(90.0)$ & $96.7(83.9)$ & $4.27(2)$ & $0.978(2)$ \\
\hline $6.2 \times 10^{5}$ & 16.1 & 7.6 & $96.7(83.9)$ & $96.3(83.9)$ & $4.08(4)$ & $0.965(3)$ \\
\hline $6.2 \times 10^{6}$ & 15.6 & 7.9 & $96.7(83.9)$ & $96.7(83.9)$ & $4.11(3)$ & $0.963(4)$ \\
\hline $6.2 \times 10^{7}$ & 15.5 & 8.0 & $93.3(77.7)$ & $93.3(77.7)$ & $3.97(6)$ & $0.944(6)$ \\
\hline $6.2 \times 10^{8}$ & 15.6 & 7.9 & $66.7(54.8)$ & $66.7(54.8)$ & $2.84(7)$ & $0.813(7)$ \\
\hline Water $(0.05$ percent Tween & 15.1 & 7.5 & $100.0(90.0)$ & $100.0(90.0)$ & $4.42(1)$ & $1.000(1)$ \\
\hline \multicolumn{7}{|l|}{ 80) } \\
\hline$P=0.05$ & 0.5 & 0.6 & $8.5(14.3)$ & $9.3(15.9)$ & & \\
\hline \multicolumn{7}{|l|}{ Topical Application } \\
\hline $6.2 \times 10^{3}$ & 15.4 & 7.7 & $100.0(90.0)$ & $100.0(90.0)$ & $4.33(1)$ & $1.01(1)$ \\
\hline $6.2 \times 10^{4}$ & 16.0 & 7.7 & $100.0(90.0)$ & $100.0(90.0)$ & $4.22(4)$ & $1.00(2)$ \\
\hline $6.2 \times 10^{5}$ & 15.5 & 7.8 & $100.0(90.0)$ & $100.0(90.0)$ & $4.29(2)$ & $1.00(2)$ \\
\hline $6.2 \times 10^{6}$ & 15.9 & 7.8 & $96.7(83.9)$ & $96.7(83.9)$ & $4.08(5)$ & $1.00(2)$ \\
\hline $6.2 \times 10^{7}$ & 16.0 & 7.9 & $86.7(68.9)$ & $86.7(68.9)$ & $3.63(6)$ & $0.93(3)$ \\
\hline $6.2 \times 10^{8}$ & 15.4 & 7.8 & $70.0(56.8)$ & $70.0(56.8)$ & $3.02(7)$ & $0.86(4)$ \\
\hline Water $(0.05$ percent Tween & 15.5 & 7.9 & $100.0(90.0)$ & $100.0(90.0)$ & $4.27(3)$ & $1.00(2)$ \\
\hline \multicolumn{7}{|l|}{ 80) } \\
\hline$P=0.05$ & 0.6 & 0.8 & $5.4(7.7)$ & $5.4(7.7)$ & & \\
\hline
\end{tabular}

Data in parentheses are angular transformed except for Growth and Success indices which indicate ranking 
Table.8 Developmental behaviour of S. litura when 0-24 h old larvae crawled on castor for leaves treated with different spore Concentrations and topical application of Delhi isolate of B. bassiana

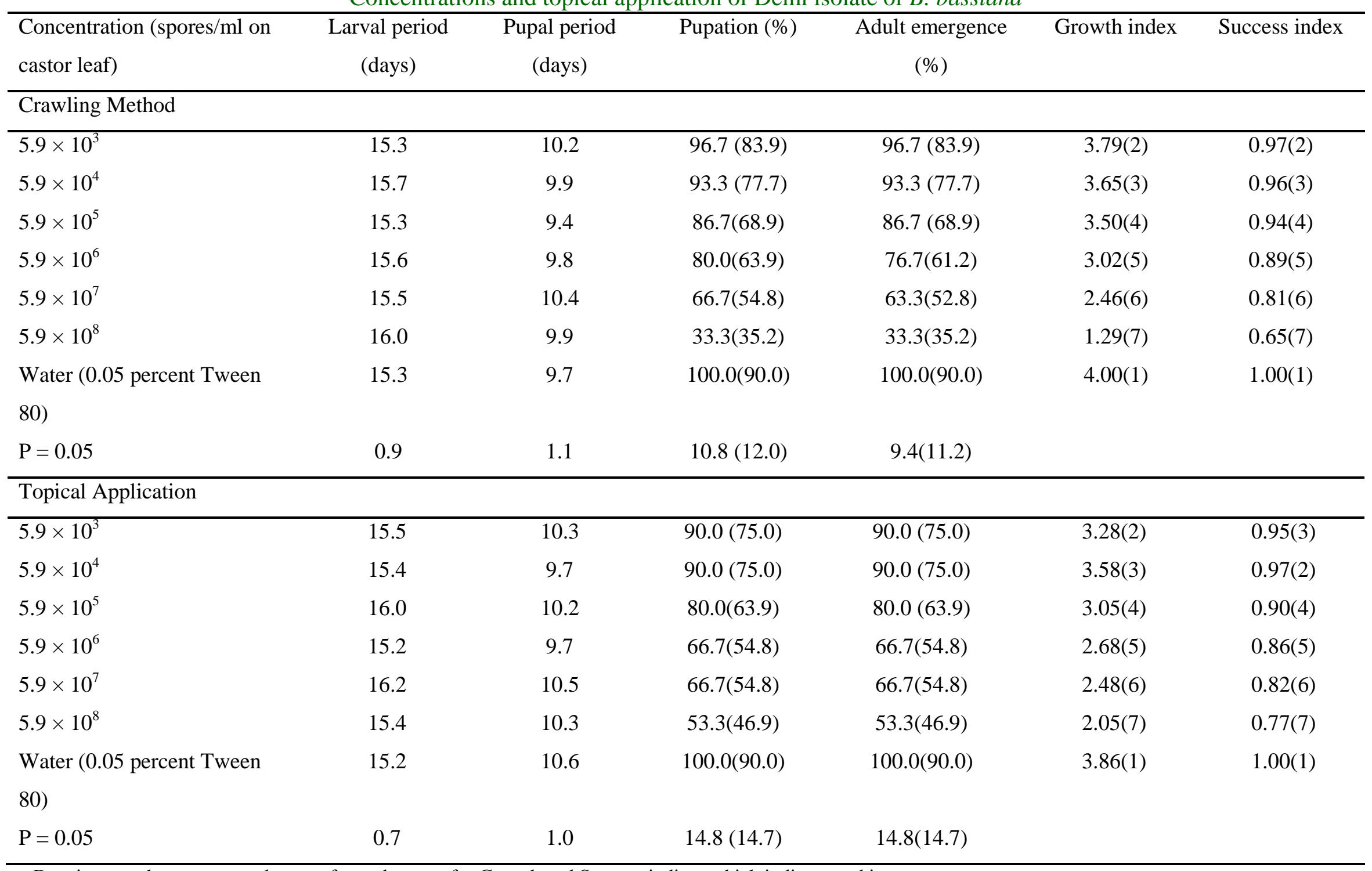

Data in parentheses are angular transformed except for Growth and Success indices which indicate ranking 
Table.9 Developmental behaviour of $S$. litura when 6 day old larvae crawled on castor for leaves treated with different spore

Concentrations of Delhi isolate of $B$. bassiana

\begin{tabular}{|c|c|c|c|c|c|c|}
\hline $\begin{array}{l}\text { Concentration (spores/ml on } \\
\text { castor leaf) }\end{array}$ & $\begin{array}{l}\text { Larval period } \\
\text { (days) }\end{array}$ & $\begin{array}{l}\text { Pupal period } \\
\text { (days) }\end{array}$ & Pupation (\%) & $\begin{array}{c}\text { Adult emergence } \\
\qquad \%)\end{array}$ & Growth index & Success index \\
\hline \multicolumn{7}{|l|}{ Crawling Method } \\
\hline $5.9 \times 10^{4}$ & 14.3 & 8.6 & $100.0(90.0)$ & $100.0(90.0)$ & $4.40(3)$ & $0.98(3)$ \\
\hline $5.9 \times 10^{5}$ & 14.3 & 8.4 & $96.7(83.9)$ & $96.7(83.9)$ & $4.25(4)$ & $0.95(5)$ \\
\hline $5.9 \times 10^{7}$ & 14.3 & 8.5 & $93.3(81.1)$ & $90.0(81.1)$ & $3.94(6)$ & $0.94(6)$ \\
\hline $5.9 \times 10^{8}$ & 14.4 & 8.4 & $86.7(68.9)$ & $86.7(68.9)$ & $3.80(7)$ & $0.91(7)$ \\
\hline Water $(0.05$ percent Tween 80$)$ & 14.4 & 7.7 & $100.0(90.0)$ & $100.0(90.0)$ & $4.52(1)$ & $1.00(1)$ \\
\hline$P=0.05$ & 0.4 & 0.9 & $10.1(14.6)$ & $10.1(14.6)$ & & \\
\hline $5.9 \times 10^{5}$ & 14.2 & 8.4 & $100.07(90.0)$ & $100.0(90.0)$ & $4.43(2)$ & $1.02(1)$ \\
\hline $5.9 \times 10^{6}$ & 14.1 & 8.0 & $93.3(77.7)$ & $93.3(77.7)$ & $4.22(5)$ & $1.02(1)$ \\
\hline $5.9 \times 10^{7}$ & 14.2 & 8.5 & $93.3(77.7)$ & $90.0(74.9)$ & $3.98(6)$ & $0.98(5)$ \\
\hline $5.9 \times 10^{8}$ & 14.7 & 8.5 & $86.7(68.9)$ & $86.7(68.9)$ & $3.74(7)$ & $0.93(6)$ \\
\hline Water $(0.05$ percent Tween & 14.6 & 8.3 & $100.0(90.0)$ & $100.0(90.0)$ & $4.39(3)$ & $1.00(3)$ \\
\hline \multicolumn{7}{|l|}{ 80) } \\
\hline $\mathrm{P}=0.05$ & 0.3 & 0.8 & $6.6(10.4)$ & $8.5(11.9)$ & & \\
\hline
\end{tabular}

Data in parentheses are angular transformed except for Growth and Success indices which indicate ranking 


\section{Chikmagalore isolate}

A gradual decrease in larval survival with increase in spore concentration of Chikmagalore isolate was recorded by crawling method on neonate larvae of $S$. litura (Figure 5).

Larval and pupal periods were somewhat similar in all test concentrations but pupation and adult emergence were significantly lower at $6.2 \times 10^{8}$ spores per ml (Table 6).

Figure 6 reveals that Chikmagalore isolate effectively reduced the survival of neonate larvae upto 46.7 percent when $6.2 \times 10^{8}$ spores per $\mathrm{ml}$ were applied topically. In rest of the concentrations survival ranged from 80.0 to 93.3 percent. This isolate also failed to show any adverse effect on developmental period of $S$. litura when topically applied on neonate larvae (Table 6).

Pupation and adult emergence were 43.3 percent at $6.2 \times 10^{8}$ spores per $\mathrm{ml}$, while in other concentrations adult emergence ranged from 76.7 to 90.0 percent while in control 100 percent pupation and adult emergence were recorded.

Test with 6 day old larvae of $S$. litura when sprayed with $6.2 \times 10^{8}$ spores per ml topically or when allowed to crawl on treated leaves with above concentration of spores resulted in a marked reduction in larval survival. Pupal periods were not affected by this isolate by both methods of applications (Table 7). Adult emergence ranged from 66.7 to 96.7 percent by crawling method while it was 70.0 to 100 percent by topical application. However, Growth index were 2.84 to 3.97 and 3.02 to 3.63 while Success index were 0.86 to 0.93 and 0.86 to 0.93 at $6.2 \times 10^{7}$ and $6.2 \times 10^{8}$ spores per ml when both the methods of application were used indicating poor performance of this isolate (Table 7).

\section{Delhi isolate}

Figure 7 reveals that larval survival decreased with increase in concentration of spores from $5.9 \times 10^{3}$ to $5.9 \times 10^{7}$ spores per ml of Delhi isolate. However, a sudden fall in survival was observed at $5.9 \times 10^{8}$ spores per $\mathrm{ml}$ applied by crawling method on neonate larvae of S. litura. Pupation and adult emergence were 33.3 percent at $5.9 \times 10^{8}$ spores per $\mathrm{ml}$ (Table 8) Growth and Success indices were 2.05 and 0.77 , respectively, when this level of spores were applied on insects.

Mortality of neonate larvae started 2 days after topical application of Delhi isolate and progressed upto 10 days (Figure 8). Adult emergence ranged from 53.3 to 90.0 percent at $5.9 \times 10^{5}$ to $5.9 \times 10^{8}$ spores per ml of Delhi isolate applied topically on neonate larvae (Table 8).

Application of $5.9 \times 10^{8}$ spores per $\mathrm{ml}$ of Delhi isolate on 6 days old larvae by two methods revealed less effect as reduction of larval survival was 13.3 percent. This isolate failed to show any adverse effect on larval and pupal periods by either methods of application (Table 9). Pupation and adult emergence were also not affected by this isolate. Similar response was also recorded on Growth and Success indices. Ndoye (1977) reported 30 to 75 percent larval mortality of C. suppressalis by different isolates of $B$. bassiana. However, 77.5 to 87.5 percent mortality was recorded on third instar larvae of E. ello by B.b 9601 isolate of this fungus (Munera et al., 1999).

Therefore, it is apparent that B. bassiana did not affect the developmental period when neonate and 6 day old larvae were sprayed with spores either directly on insects or by allowing the larvae to crawl over the sprayed castor leaves. However, application of $10^{3}$ to $10^{8}$ spores per $\mathrm{ml}$ of all isolates of $B$. bassiana 
on neonate larvae by both methods of application indicated a decrease in larval survival and, percent pupation and adult emergence. On the other hand larval survival of 6 day old larvae was low when $10^{8}$ spores per $\mathrm{ml}$ for all isolates were applied by both methods. Therefore, it is suggested that $B$. bassiana spores should be used on the neonate larvae of $S$. litura for achieving an effective control of this pest.

\section{References}

Prasad, J. and Bhattacharya, A. K. 1975. Growth and development of Spodoptera littoralis (Boisd.) on several plants. Z. angew. Ent, 79: 34-48.

Bhattacharya, A.K. and Rathore, Y.S. 1997. Survey and study of the bionomics of major soybean insects and their chemical control. Pantnagar, Nainital, India, G.B. Pant University of Agriculture and Technology. 324 p.

Seema Rani, Goel, B.B. and Gupta, G.P. 2002. Growth and development of Spodoptera litura Fabricius on different host plants. Ann. Pl. Prot. Sci., 16: 216219.

Krishnaiah, K., Ramakrishnan, N. and Reddy, P.G. 1983. Control of tobacco caterpillar Spodoptera litura (F.) on black gram by nuclear polyhedrosis virus. Paper presented at Annual Workshop on Coordinated Pulse Improvement Project, Pune, April, 1983.

Ramakrishnan, N., Saxena, V.S. and Dhingra, S. 1984. Insecticide resistance in the population of Spodoptera litura (F.) in Andhra Pradesh. Pesticides, 18: 23-27.

Murugesan, K. and Dhingra, S. 1995. Variability in resistance pattern of various group of insecticides evaluated against Spodoptera litura (Fabriciles) during a period spanning over three decades. J. Ent. Res., 19: 313-319.

Jayraj, S. 1985. Microbial control and pest management. Coimbtore, India, Tamilnadu Agricultural University, 278 p.

Ferron, P. 1985. Fungal control. In: Comprehensive Insect Physiology, Biochemistry and Pharmacology. Vol. XII. (Eds.) G. A. Kerkut and L.I. Gilbert, ed. Pergamon Press, New York. pp. 313 - 346.

Pant, N.C. 1956. Nutritional studies on Trogoderma granarium Everts. Indian J. Ent., 18: 266-268.

Ndoye, M. 1977. The susceptibility of larvae and pupae of Chilo suppressalis Walker to various entomopathogenous fungi (Fungi Imperfecti), and the factors that determine susceptibility to the mycosis caused by Beauveria bassiana (Bals.) Vuill. Bull. Inst. Fond. Afrique Noire, 39: $303-317$

Munera, S.D.F., Rios, T.J. de los, Bellotti, A.C. and de los, Rios, T.J. 1999. Pathogenicity of Erinnyis ello (Lepidoptera: Sphingidae) in laboratory conditions of entomopathogenic fungus collected in commercial crops of cassava, Manihot esculenta, in Cauca Valley, Colombia. Rev. Col. Entomol., 25: 3 - 4 .

\section{How to cite this article:}

Harikesh Singh and Wajid Hasan. 2017. Detrimental Effects of Different Isolates of Beauveria bassiana on Developmental Profile of Spodoptera litura. Int.J.Curr.Microbiol.App.Sci. 6(7): 2378-2394. doi: https://doi.org/10.20546/ijcmas.2017.607.340 